ORNL/TM-2008/085

\title{
A Vertical Grid Module for Baroclinic Models of the Atmosphere
}

\author{
June 2008
}

John B. Drake 


\section{DOCUMENT AVAILABILITY}

Reports produced after January 1, 1996, are generally available free via the U.S. Department of Energy (DOE) Information Bridge:

Web Site: http://www.osti.gov/bridge

Reports produced before January 1, 1996, may be purchased by members of the public from the following source:

National Technical Information Service

5285 Port Royal Road

Springfield, VA 22161

Telephone: 703-605-6000 (1-800-553-6847)

TDD: 703-487-4639

Fax: 703-605-6900

E-mail: info@ntis.fedworld.gov

Web site: http://www.ntis.gov/support/ordernowabout.htm

Reports are available to DOE employees, DOE contractors, Energy Technology Data Exchange (ETDE), and International Nuclear Information System (INIS) representatives from the following sources:

Office of Scientific and Technical Information

P.O. Box 62

Oak Ridge, TN 37831

Telephone: $865-576-8401$

Fax: 865-576-5728

E-mail: reports@adonis.osti.gov

Web site: http://www.osti.gov/contact.html

This report was prepared as an account of work sponsored by an agency of the United States Government. Neither the United States nor any agency thereof, nor any of their employees, makes any warranty, express or implied, or assumes any legal liability or responsibility for the accuracy, completeness, or usefulness of any information, apparatus, product, or process disclosed, or represents that its use would not infringe privately owned rights. Reference herein to any specific commercial product, process, or service by trade name, trademark, manufacturer, or otherwise, does not necessarily constitute or imply its endorsement, recommendation, or favoring by the United States Government or any agency thereof. The views and opinions of authors expressed herein do not necessarily state or reflect those of the United States Government or any agency thereof. 
ORNL/TM-2008/085

\title{
A VERTICAL GRID MODULE FOR BAROCLINIC MODELS OF THE ATMOSPHERE
}

\author{
John B. Drake
}

Date Published: June 2008

Prepared by OAK RIDGE NATIONAL LABORATORY

P. O. Box 2008

Oak Ridge, Tennessee 37831-6285

managed by UT-Battelle, LLC

for the

U. S. DEPARTMENT OF ENERGY

under contract DE-AC05-00OR22725 



\section{Contents}

List of Figures $\quad$ v

Executive Summary vii

$\begin{array}{ll}\text { Abstract } & \text { ix }\end{array}$

1 Introduction 1

2 Generalized Meteorological Vertical Coordinates 2

2.1 Variables . . . . . . . . . . . . . . . 2

3 Lorentz Vertical Discretization 3

3.1 Lorentz vertical grid module . . . . . . . . . . . . . . 4

3.2 Geopotential and the Discrete Hydrostatic Equation . . . . 5

3.3 Thermal Wind . . . . . . . . . . . . . . . 6

3.4 Vertical Advection . . . . . . . . . . . . . . 7

3.5 Vertical Mass Flux . . . . . . . . . . . . . . . 7

3.6 Vertical Integral Terms . . . . . . . . . . . . . 8

4 Test Methods $\quad 8$

4.1 Vertical Structure Equation . . . . . . . . . . . . . 8

4.2 Baroclinic Right Hand Sides . . . . . . . . . . . . . . . 15

$\begin{array}{llr}5 & \text { Conclusion } & 17\end{array}$

$\begin{array}{llr}6 & \text { Acknowledgments } & 18\end{array}$

$\begin{array}{ll}\text { A The } 1976 \text { Standard Atmosphere } & 18\end{array}$

B Compact Vertical Discretization based on Laguerre Polynomials 18 



\section{List of Figures}

1 Lorentz Vertical Grid with Location of Discrete Variables . . 4

2 Lorentz Vertical Column representing a reference atmosphere with $p_{\text {top }}=2.8 \mathrm{hPa} \ldots \ldots \ldots \ldots \ldots$

3 Normal modes of the vertical structure equation with $p_{\text {top }}=$ $2.9 \mathrm{hPa}$ and $K=26$ and 96 . Only the six largest modes (excluding the external mode), and their equivalent depths are displayed. Equivalent depths $(\mathrm{m})$ are normalized so that the largest mode has a depth of $10 \mathrm{~km} \ldots$. . . . . . . . . 14

$4 \quad$ Equivalent depths for $p_{t o p}=2.9 h P a$ and $K=96$. . . . . 15

5 Computational Modes for the Lorentz grid $p_{t o p}=2.9 \mathrm{hPa}$ and $K=96 . \ldots \ldots \ldots \ldots \ldots \ldots$

6 Standard Atmosphere Temperature Profile . . . . . . . . . . 20

7 Stencil for Charney-Phillips grid with fourth order compact method. . . . . . . . . . . . . . 21

8 Hydrostatic solution for the geopotential using fourth order compact method $p_{t o p}=2.8 \mathrm{hPa}$ and $K=26$. The standard atmosphere temperature profile is on a Charney-Phillips grid of equally spaced points in $\sigma \ldots \ldots \ldots . \ldots . \ldots 23$

9 Relative error in geopotential $p_{\text {top }}=0.1 \mathrm{hPa}$ and $K=9,18,36,72,144,288,576$ on a Charney-Phillips grid of equally spaced points in $\sigma$. . . 24 



\section{Executive Summary}

The vertical grid of an atmospheric model assigns dynamic and thermodynamic variables to grid locations. The vertical coordinate is typically not height but one of a class of meterological variables that vary with atmospheric conditions. The grid system is chosen to further numerical approximations of the boundary conditions so that the system is terrain following at the surface. Lagrangian vertical coordinates are useful in reducing the numerical errors from advection processes. That the choices will effect the numercial properties and accuracy is explored in this report. A MATLAB class for Lorentz vertical grids is described and applied to the vertical structure equation and baroclinic atmospheric circulation. A generalized meteorolgoical coordinate system is developed that can support $\sigma$, isentropic $\theta$ vertical coordinate, or Lagrangian vertical coordinates. The vertical atmospheric column is a MATLAB class that includes the kinematic and thermodynamic variables along with methods for computing geopoentials and terms relevant to a 3D baroclinc atmospheric model. 



\begin{abstract}
The vertical grid of an atmospheric model assigns dynamic and thermodynamic variables to grid locations. The vertical coordinate is typically not height but one of a class of meterological variables that vary with atmospheric conditions. The grid system is chosen to further numerical approximations of the boundary conditions so that the system is terrain following at the surface. Lagrangian vertical coordinates are useful in reducing the numerical errors from advection processes. That the choices will effect the numercial properties and accuracy is explored in this report. A MATLAB class for Lorentz vertical grids is described and applied to the vertical structure equation and baroclinic atmospheric circulation. A generalized meteorolgoical coordinate system is developed which can support $\sigma$, isentropic $\theta$ vertical coordinate, or Lagrangian vertical coordinates. The vertical atmospheric column is a MATLAB class that includes the kinematic and thermodynamic variables along with methods for computing geopoentials and terms relevant to a 3D baroclinc atmospheric model.
\end{abstract}





\section{Introduction}

Generalized meteorological coordinates were first explored with isentropic coordinate models introduced for numerical weather prediction in the 1970's $[12,3]$. The attraction of the isentropic coordinate model was largely theoretical because for adiabatic flow the isentropic surfaces are material surfaces. Given the low resolution of atmospheric models, a higher degree of accuracy could be expected for the advective terms with an isentropic formulation.

Significant problems with the handling of the lower boundary condition and its implication for conservation and boundary layer approximations were addressed by the introduction of a hybrid (patched) model [34]. By use of $\sigma$ coordinates through the boundary layer, the lower boundary condition problem was moved to the top of the boundary layer, where isentropic coordinates started. Conservation and consistency conditions were imposed on the seam between sigma and free atmosphere. This formulation still required massless layers in the isentropic domain and because of the special care necessary in dealing with these layers, use of isentropic coordinate models was never widespread.

Isentropic analysis of global weather patterns and circulations continued to develop and provide a complementary understanding to the isobaric analysis $[33,17]$. The effects of heating on the circulation are most clearly seen with the isentropic analysis. Heating forces the motion of isentropic surfaces upward indicating the vertical mass flux which marks the global circulations of the Hadley and Ferrel cells along with monsoonal variations. The differential heating of the atmosphere drives a mass circulation whose function is to transport energy from heat sources to heat sinks. This analysis has become important for the understanding of regional weather patterns and climate.

A comparison of an isentropic coordinate model with a sigma models suggested that the transport properties of the isentropic model may be important for simulated precipitation and tracer distributions [16, 37]. While these studies are relevant for weather time scale circulations, another study suggests the relevance of isentropic coordinate models for climate modeling [18]. Johnson argues that the cold bias at the poles in nearly all climate models is an artifact of their formulation. "The cold temperature bias develops to provide for the increased sink of entropy by infrared cooling that is necessary to offset the positive definite aphysical source of entropy from mixing by numerics and the inadequacies of parameterization." ([18, p.5]) For a climate model to be without drift and to be "correct" for coupling with other 
earth system component models energy conservation should be satisfied as well as the entropy principle. The claim is that spurious entropy sources can be largely eliminated by choice of an isentropic coordinate sytsem, since this carefully separates the mixing of energy from vertical advection, and by choice of potential temperature as the thermodynamic variable.

Recently, the formulation of isentropic coordinate models has become combined with the continuous (not patched) generalized meteorological coordinate systems introduced in [19, 15]. The paper of Konor and Arakawa [22] gives a formulation in which the vertical coordinate transitions smoothly between a sigma coordinate near the earths surface to the isentropic coordinate in the free atmosphere. This formulation has several significant advantages over earlier work. Foremost is the elimination of massless layers at the surface and the need to patch coordinates at a seam. The conservation principles can be developed in the generalized coordinate system without an arbitrary patch or seam being present.

Lagrangian vertical systems have also received much attention with the use of the Lin-Rood dynamics [26, 25] in several modeling systems. The Lagrangian coordinate requires a remapping every so often (at the physics timestep) to restore the original resolution and keep the mesh from developing massless layers.

The discretization of the vertical is a matter of considerable study with an ongoing debate about whether the Lorentz staggering is as good as that introduced by Charney and Phillips [4] [30]. Recently, the U.K. MetCenter has developed a atmospheric model based on the Charney-Phillips vertical grid and their diagnosis of errors is very interesting. This relates to an old issue concerning the normal modes of the atmosphere and the fact that Charney's original argument was flawed in the treatment of a (singular) Sturm-Liouville problem.

The algorithmic choices for the treatment of vertical terms have important implications for the required vertical resolution of atmospheric models [36] and the treatment the upper boundary condition as applied in the vertical coordinate system. Some of these issues are explored in this report with the intention of providing tools and a starting point for other research projects.

\section{Generalized Meteorological Vertical Coordinates}

\subsection{Variables}

The variables we will be concerned with are the 
- vertical coordinate $(\zeta)$,

- vertical coordinate time derivative $(\dot{\zeta})$,

- $\operatorname{pseudo}$ density $\left(\pi=\frac{\partial p}{\partial \zeta}\right)$,

- $\operatorname{pressure}(p)$,

- surface pressure $\left(p_{s}\right)$,

- temperature $(T)$,

- potential temperature $\left(\theta \equiv \frac{C_{p} T}{\Pi}\right)$,

- horizontal velocity (v).

- horizontal divergence $\left(\delta=\nabla_{\zeta} \cdot \mathbf{v}\right)$,

- horizontal potential vorticity $(\eta=\xi+f)$,

- vertical pressure velocity $\left(\omega=\frac{D p}{D t}\right)$,

- pressure advection $\left(\hat{\omega}=\dot{\zeta} \frac{\partial p}{\partial \zeta}=\dot{\zeta} \pi\right)$,

- geopotential $(\Phi)$,

- Montgomery potential $\left(M \equiv c_{p} T+\Phi\right)$.

- static stability $\left(\gamma=-\frac{T}{\theta} \frac{\partial \theta}{\partial p}\right)$.

- Exner function $\left(\Pi \equiv c_{p}\left(\frac{p}{p_{0}}\right)^{\kappa}\right)$,

We will write the generalized vertical coordinate $\zeta$. The horizontal coordinates will not be of concern as we will use the device of writing operators in horizontal and vertical form.

\section{Lorentz Vertical Discretization}

The particular organization we will use of the discrete variables staggered between half and full levels of the grid is called the Lorentz grid. An alternative staggering and location of the vertical grid is the Charney-Phillips grid [30]. The Lorentz grid is used in the Community Atmospheric Model (CAM3) [6] and this vertical grid module should be compatible with the CAM discretizations using a hybrid $\sigma-p$ vertical coordinate. The cell centers (layers) will be indexed from 1 to $K$ with half levels (cell edges) indexed 
with half value indices from $1 / 2$ to $K+1 / 2$. A pictorial representation of the Lorentz vertical grid is given in Figure (1). Thermodynamic and horizontal momentum variables are located at cell centers (integer indices) while the vertical momenum and pressure variables are located at half index edges.
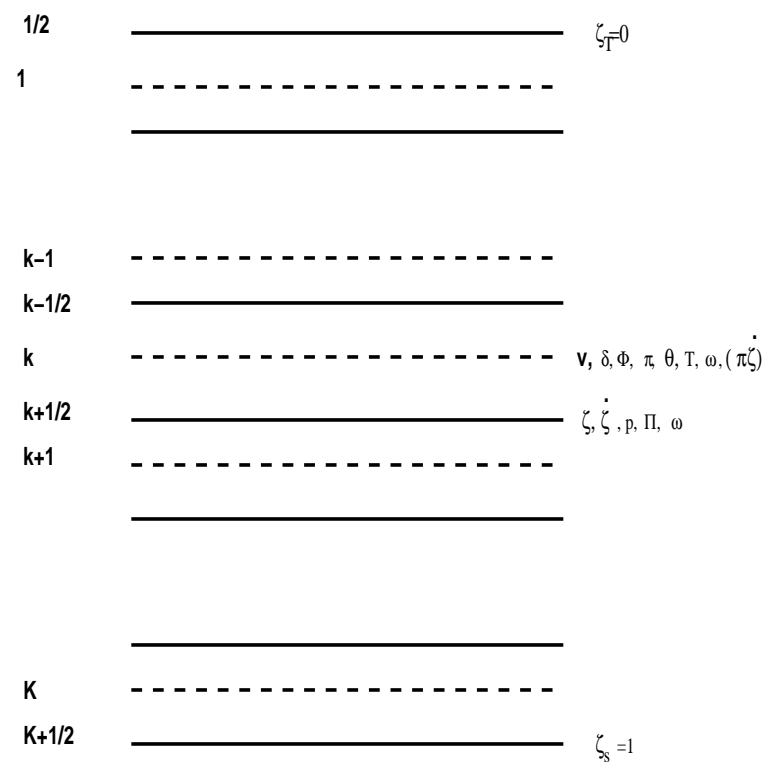

Figure 1: Lorentz Vertical Grid with Location of Discrete Variables

\subsection{Lorentz vertical grid module}

The MATLAB class lvgrid sets up a Lorentz vertical grid. The methods of this class include the obligatory get and set so that the default values can be over-ridden. The grid class contains only the $\zeta$ and $\dot{\zeta}$ variables and routines that calculate vertical integrals and advection terms that utilize the discrete coordinates.

The variables and dynamical terms involving the vertical of a baroclinic atmosphere are provided in the Lorentz vertical column class, lvcolumn. The thermodynamic variables, $\ln \pi, \theta, T$ along with kinematic variables $\eta, \delta, \mathbf{v}$ are data of the class. Other values are calculated from diagnostic relations based on the prognostic inputs of the column. For example, the geopotential $\Phi$ is calculated based on the half-index values of the pressure, $p$ and the integer-index values of the temperature, $T$. 
Methods of the lvcolumn class compute diagnostic variables using discrete diagnostic relationships and terms of the mass, momentum and energy equations. The hydrostatic assumption is made for the vertical momentum equation and the geopotential is computed from this. Diagnostic relations are updated in the update method of the lvcolumn class. The terms that are approximated from the continuity and momentum equations are $\frac{\partial(\dot{\zeta} \pi)}{\partial \zeta}, \dot{\zeta} \frac{\partial \psi}{\partial \zeta}$, $\omega$ and $\hat{\omega}$. The right hand side terms of a baroclinic model are calculated in the lvterms method.

\subsection{Geopotential and the Discrete Hydrostatic Equation}

The hydrostatic equation written in a generalized coordinate is

$$
\frac{\partial \Phi}{\partial \zeta}=\frac{R T}{p} \frac{\partial p}{\partial \zeta}
$$

and in pressure coordinate

$$
\frac{\partial \Phi}{\partial \ln p}=-R T
$$

Integrating, the hydrostatic equation can be written as

$$
\Phi(\zeta)=\Phi_{s}+R \int_{p(\zeta)}^{p_{s}} T d \ln p
$$

The discretization of the hydrostatic relation introduces the geopotential and a hydrostatic matrix (see CAM-SLD equation (3.264) [7]),

$$
\Phi_{k}=\Phi_{s}+R \sum_{l=k}^{K} H_{k l} T_{l}
$$

where $H_{k l}=\int_{p\left(\zeta_{l+1 / 2}\right)}^{p\left(\zeta_{l-1}\right)} d \ln p$ for $l \geq k$ and 0 , otherwise. Similarly, for terms in the energy equation

$$
\int_{p_{\text {top }}}^{p(\zeta)} \delta d p=\sum_{l=1}^{k} D_{l k} \delta_{l}
$$

where $D_{l k}=\int_{p\left(\zeta_{l+1 / 2}\right)}^{p\left(\zeta_{l-1 / 2}\right)} d p$ for $l \leq k$ and 0 , otherwise.

Remark: The approximation of the hydrostatic relation in the vertical determines many of the numerical properties of the model. If a higher 
order numerical approximation is desired, this can be formulated using basis functions $\left\{\psi_{l}\right\}$ to represent the temperature. Let

$$
T(p)=\sum_{l} T_{l} \psi_{l}(p)
$$

The $T_{l}$ must now be interpreted as node values with the Lorentz grid cell edges thought of as B-Spline knots, for example. Substituting into the hydrostatic relation,

$$
\Phi(\zeta)=\Phi_{s}+R \sum_{l} T_{l} \int_{p(\zeta)}^{p_{s}} \psi_{l}(p) d \ln p
$$

This is the same form regardless of the choice of the basis functions with $H_{k l}=\int_{p\left(\zeta_{l+1 / 2}\right)}^{p\left(\zeta_{l-1 / 2}\right)} \psi_{l}(p) d \ln p$. For a linear B-Spline basis, the standard vertical discretization is obtained. A compact method for the hydrostatic equation using Laguerre polynomials is described in appendix B.

\subsection{Thermal Wind}

The accuracy of the vertical discretization will be partially determined by how well the discretization approximates the dominant terms coming from the hydrostatic equation and the thermal wind relationship.

The thermal wind relationship (in pressure coordinates) is derived from the hydrostatic relation [[14], p. 70] and can be expressed,

$$
f \mathbf{k} \times \frac{\partial \mathbf{v}}{\partial \ln p}=-R \nabla_{p} T
$$

The vertical discretization at a level $k$ follows the discretization of the hydrostatic equation so

$$
\mathbf{v}_{k}=\mathbf{v}_{s}+\frac{R}{f} \sum_{l=k}^{K} H_{k l}\left(\mathbf{k} \times \nabla_{p} T_{l}\right) .
$$

The thermal wind equation shows how closely coupled the horizontal flow is with the vertical structure through the hydrostatic approximation. Temperature gradient approximation and the hydrostatic approximation couple multiple levels through this equation. 


\subsection{Vertical Advection}

The difference scheme for advection in the vertical should conserve momentum and kinetic energy (or vorticity and enstrophy) as per Arakawa. We consider advection schemes that conserve $\psi$ and $\psi^{2}$, in the equation

$$
\frac{\partial \psi}{\partial t}+\dot{\zeta} \frac{\partial \psi}{\partial \zeta}=0
$$

On the Lorentz grid $\dot{\zeta}$ is located on cell edges. The vertical discretization that conserves $\psi$ and $\psi^{2}$ is

$$
\left(\dot{\zeta} \frac{\partial \psi}{\partial \zeta}\right)_{k}=\frac{1}{2\left(\zeta_{k+1 / 2}-\zeta_{k-1 / 2}\right)}\left[\dot{\zeta}_{k+\frac{1}{2}}\left(\psi_{k+1}-\psi_{k}\right)+\dot{\zeta}_{k-\frac{1}{2}}\left(\psi_{k}-\psi_{k-1}\right)\right] .
$$

For fields located on the integer index layers, the lvgrid class method that computes the advection term is kadvect. For fields located at the half index edges, the method is hadvect.

The vertical advection term in the $\zeta$ coordinate can also be expressed in terms of pressure as

$$
\dot{\zeta} \frac{\partial}{\partial \zeta}=-m \dot{\zeta} \frac{\partial}{\partial p}=\hat{\omega} \frac{\partial}{\partial p} .
$$

where

$$
\hat{\omega}=\dot{\zeta} \frac{\partial p}{\partial \zeta}=\dot{\zeta} \pi
$$

The $\hat{\omega}$ is computed as a diagnostic relation in the lvcolumn class update method.

\subsection{Vertical Mass Flux}

The vertical mass flux term that appears in a continuity equation is

$$
\frac{\partial}{\partial \zeta}(\dot{\zeta} \pi)
$$

The $k$-th layer approximation is

$$
\left(\frac{\partial}{\partial \zeta}(\dot{\zeta} \pi)\right)_{k}=\frac{1}{2\left(\zeta_{k+1 / 2}-\zeta_{k-1 / 2}\right)}\left[\dot{\zeta}_{k+1 / 2}\left(\pi_{k+1}+\pi_{k}\right)-\dot{\zeta}_{k-1 / 2}\left(\pi_{k}+\pi_{k-1}\right)\right] .
$$

The method vflux of @lvgrid computes this term. 


\subsection{Vertical Integral Terms}

The energy equation involves $\omega$, the vertical pressure velocity, which can be expressed using the continuity equation. We have

$$
\omega \equiv \frac{D p}{D t}=\mathbf{v} \cdot \nabla_{\zeta} p-\int_{\zeta_{t o p}}^{\zeta} \nabla_{\zeta} \cdot(\pi \mathbf{v}) d \zeta .
$$

This is written as

$$
\omega=\mathbf{v} \cdot \nabla_{\zeta} p-\int_{\zeta_{t o p}}^{\zeta} \mathbf{v} \cdot \nabla_{\zeta} \pi d \zeta-\int_{p_{t o p}}^{p(\zeta)} \delta d p
$$

The integrals appearing in this expression are computed using the lvgrid methods vintz and vintp with the rectangle rule

$$
\begin{aligned}
\int_{\zeta_{t o p}}^{\zeta_{k+1 / 2}} \psi d \zeta & =\sum_{l=1}^{k} \psi_{l}\left(\zeta_{l+1 / 2}-\zeta_{l-1 / 2}\right), \\
\int_{p_{t o p}}^{p\left(\zeta_{k+1 / 2}\right)} \delta d p & =\sum_{l=1}^{k} \delta_{l}\left(p_{l+1 / 2}-p_{l-1 / 2}\right) .
\end{aligned}
$$

\section{Test Methods}

Unit tests that exercise the lvcolumn and lvgrid classes are included in the test method of lvcolumn and VSE that solves the vertical structure equation. In addition to checking the implementation these tests also illustrate the use of the module and its approximations.

\subsection{Vertical Structure Equation}

A separation of variables technique applied to the 3-D baroclinic equations leads to consideration of the vertical modes of the atmosphere $[1,20]$. The vertical structure equation (VSE) is an example of the use of the vertical discretization, and a test of the numerics of the Lorentz grid. The vertical structure function $Z$ may also be derived from the barotropic vorticity equation in 3-D [29] [9]. The equation for $Z$, in terms of pressure coordinates, is

$$
\frac{d}{d p}\left(\frac{p}{R \gamma} \frac{d Z}{d p}\right)+\frac{Z}{g H}=0
$$


with boundary conditions

$$
\begin{aligned}
p \frac{d Z}{d p} & =0, \quad \text { at } p=p_{t o p} \\
\frac{d Z}{d p}+\frac{\gamma}{T_{s}} Z & =0, \quad \text { at } \quad p=p_{s} .
\end{aligned}
$$

The parameters are $R$ the ideal gas constant, $g$ is the gravitational acceleration, $\gamma$ the static stability parameter with $\gamma=-\frac{T}{\theta} \frac{\partial \theta}{\partial p}$. $H$ is referred to as the eigenvalue of equivalent depth (and will be found as part of the solution of the eigenanalysis). The thermodynamic variables in the atmospheric column are $T$ temperature and $\theta$ potential temperature.

The eigenvalue problem reveals the "normal modes" of the vertical structure. An interpretation of the implications for representing atmospheric data is given in [1]. The approach through normal modes [9] also lends itself to analysis of particular meteorological events. Their use in determining model vertical resolution is discussed in [36]. Normal modes are also used to separate and control "fast" gravity modes from the "slow" Rossby modes. Machenhauer [27] and Baer [2] first used normal mode initialization for weather models and Daley [8] suggests their use for the development of long time step integration methods.

The vertical structure equation, and the normal modes, are involved in a fundamental way in the development of turbulence theory for the atmosphere. The formulation of the boundary conditions for the vertical structure equation determines the energy closure for atmospheric flow. The closure assumptions give rise to a misconception that has followed atmospheric modeling since Charney [21]. The misconception arises because the top of the atmosphere is not a rigid boundary. Defining the vertical domain from surface to infinity, or from surface pressure to zero pressure, leads to a singular Sturm-Lioville problem [5]. Since the coefficient $\frac{p}{R \gamma}$ of the Sturm-Lioville problem vanishes as $p \rightarrow 0$ (and the $\sigma$-coordinate interval expands to $[0,1]$ ) the VSE is a singular Sturm-Liouville problem. This choice of $p_{\text {top }}=0$ correspondes to an infinite extent of the upper atmosphere. What is usually done is to set $p_{\text {top }}$ to a small positive number corresponding to a $\sigma$ interval of, for example, $[0.028,1]$. This eliminates the singularity of the Sturm-Lioville problem turning it into a regular Sturm-Lioville problem. In either case, the theory says that there exist a complete set of orthonormal modes. Unfortunately, the choice of boundary condition may lead to elimination of some modes all together. The choice made by Charney, $\frac{d Z}{d p}=0$ at the singular point, overspecifies the problem eliminating modes that potentially carry energy in the dynamic energy cascade. The correct singular Sturm-Lioville 
boundary condition is $Z$ bounded(finite) at $p_{t o p}=0$. In this case, the spectrum of the singular operator is not discrete, there is continuous spectrum associated with the infinite extent of the domain.

The numerical treatment of the spectrum is an issue for all vertical coordinate systems. The choice of a finite, rigid lid for the atmosphere, $p_{t o p}>0$ is a regularization of the problem with possibly unintended smoothing effects and certainly implications for accuracy, stability and the coupling of vertical and horizontal modes. The numerical solution of atmospheric model equations based on a discrete analog of the vertical structure equation will "exhibit" similar numerical accuracy and stability problems. The number of modes that must be resolved for correct capture of the flow energetics is considered in [1] where it is noted that some modes have a kinetic energy cascade similar to the Kolomogorov $k^{-5 / 3}$ power law and others similar to two dimensional turbulence with a $k^{-3}$ power law. (These conclusions were reached using very poorly resolved vertical modes. Only nine vertical levels were used and the choice of the model top was not investigated.)

For numerical approximation, as a rule of thumb, the number of points used in a discretization depends on the smoothness and behaviour of the function. For oscillatory functions, like the sine, 5-10 points between zeros is generally adequate to capture the qualitative behaviour of the function. A minimal parabolic fit between zeros would require three points. The $n$ th eigenfunction of the Sturm-Lioville problem has $n$ zeros in the interval [5]. Hence if $N$ modes are important in the 3-D baroclinic dynamics, then roughly $5 N$ points in the vertical discretization are called for. Deciding how many modes are needed for accuracy is a function of the choices at the top of the atmosphere boundary condition. As $p \rightarrow 0$, the oscillations of the normal modes increase near the singular boundary, see Figure 3.

This increased oscillation at the top of the atmosphere has lead to the suggestion that more points be added to resolve the structures using an unequally spaced mesh [36]. This is counter intuitive since most of the mass of the atmosphere occurs near the surface. One hopes that the stratospheric modes do not influence the near surface values very much. Unfortunately, a look at the shape of the eigenfunctions indicates otherwise. So numerics that filter these modes explicitly, by damping, or implicitly, by low resolution, may be important for reasonable results near the surface. Another option is to increase the order of the vertical discretization using basis functions, eg. finite elements or even the normal modes themselves, as illustrated in the Appendix using Laguerre polynomials in a compact vertical discretization. The vertical distribution of points on the Lorentz grid is also effected by the choice of coordinates. An isentropic coordinate distriubtion may yield a 
better distribution than a uniform $\sigma$ such as used in our test example [24].

The choice of the height for a top of the atmosphere lid used to regularize the singular problem may benefit from physical considerations. In the appendix A, height and pressure values for the standard atmosphere are given along with the thermal structure. A value near the tropopause eliminates many of the oscillatory stratospheric modes. A recent model development [11] uses a top at $10 \mathrm{~km}$ or roughly $p_{t o p}=264 \mathrm{hPa}$. Using a quadratically stretched grid with nineteen levels, the accuracy of the fifth mode is already very questionable. A leading atmospheric climate model [6] places the $p_{\text {top }}=2.9 \mathrm{hPa}$ or a height of $40 \mathrm{~km}$. The interplay between the choice of the top and the vertical discretization will be studied using the vertical structure equation with the uniform lvcolumn vertical discretization.

A control volume discretization of (Eq. 20) that follows the distribution of values on a Lorentz grid assigns $Z$ to the integer index layers, the same location as horizontal momentum and geopotential. The Lorentz vertical grid has the pressure at half index locations and pressure must be averaged to integer index locations. (This averaging is a source of discretization error and computational modes that may affect stability.) The thermodynamic parameter $\gamma$ is located at the integer index layers and values of temperature and pressure are given by the state of the atmospheric column. Integrating over a model layer, $\left[\zeta_{k-1 / 2}, \zeta_{k+1 / 2}\right]$,

$$
\begin{aligned}
& \frac{1}{\Delta p_{k}} \int_{p_{k-1 / 2}}^{p_{k+1 / 2}}\left[\frac{d}{d p}\left(\frac{p}{R \gamma} \frac{d Z}{d p}\right)+\frac{Z}{g H}\right] d p \\
= & \frac{1}{\Delta p_{k}}\left[\frac{p}{R \gamma} \frac{d Z}{d p}\right]_{p\left(\zeta_{k-1 / 2}\right)}^{p\left(\zeta_{k+1 / 2}\right)}+\frac{1}{g H} Z_{k} \\
= & \frac{1}{\Delta p_{k}}\left[\left(\frac{p_{k+1 / 2}}{R \gamma_{k+1 / 2}}\right) \frac{\left(Z_{k+1}-Z_{k}\right)}{\left(p_{k+1}-p_{k}\right)}-\left(\frac{p_{k-1 / 2}}{R \gamma_{k-1 / 2}}\right) \frac{\left(Z_{k}-Z_{k-1}\right)}{\left(p_{k}-p_{k-1}\right)}\right] \\
+ & \frac{1}{g H} Z_{k}=0 .
\end{aligned}
$$

In these equations, $\Delta p_{k}=p_{k+1 / 2}-p_{k-1 / 2}, p_{k}=\frac{1}{2}\left(p_{k+1 / 2}+p_{k-1 / 2}\right)$.

The boundary conditions are discretized at the half index surfaces by substitution into the $k=1$ layer and $k=K$ layer equations. So the top layer equation is

$$
\frac{1}{\Delta p_{1}}\left[\left(\frac{p_{3 / 2}}{R \gamma_{3 / 2}}\right) \frac{\left(Z_{2}-Z_{1}\right)}{\left(p_{2}-p_{1}\right)}-0\right]+\frac{1}{g H} Z_{1}=0
$$


The bottom layer equation is

$$
\frac{1}{\Delta p_{K}}\left[\left(-\frac{p_{s}}{T_{s} R} Z_{s}\right)-\frac{p_{K-1 / 2}}{R \gamma_{K-1 / 2}} \frac{\left(Z_{K}-Z_{K-1}\right)}{\left(p_{K}-p_{K-1}\right)}\right]+\frac{1}{g H} Z_{K}=0 .
$$

The static stability parameter is computed at half index levels as

$$
\gamma_{k+1 / 2}=-\frac{\left(T_{k}+T_{k+1}\right)}{\left(\theta_{k}+\theta_{k+1}\right)}\left(\frac{\theta_{k+1}-\theta_{k}}{p_{k+1}-p_{k}}\right)
$$

The resulting system is $\mathbf{A Z}=\lambda \mathbf{Z}$ where the eigenvalue $\lambda=\frac{-1}{g H}$ and matrix

$$
\begin{aligned}
a_{k, k-1} & =\frac{1}{\Delta p_{k}} \frac{p_{k-1 / 2}}{R \gamma_{k-1 / 2} \Delta p_{k-1 / 2}}, \\
a_{k, k} & =-\frac{1}{\Delta p_{k}}\left[\frac{p_{k+1 / 2}}{R \gamma_{k+1 / 2} \Delta p_{k+1 / 2}}+\frac{p_{k-1 / 2}}{R \gamma_{k-1 / 2} \Delta p_{k-1 / 2}}\right], \\
a_{k, k+1} & =\frac{1}{\Delta p_{k}} \frac{p_{k+1 / 2}}{R \gamma_{k+1 / 2} \Delta p_{k+1 / 2}},
\end{aligned}
$$

An eigenanalysis of the A matrix, using the MATLAB eig function, generates eigenfunctions and eigenvalues that correspond to the normal modes and the equivalent depths, $H$, for each mode. The constant function with a zero eigenvalue (infinite height), is called the external mode and is in the null space of A. A MATLAB test program, VSE.m, that solves this eigenproblem uses a sample vertical column corresponding to a reference atmosphere, Figure 2. The model top, specified by $p_{t o p}$ can be varied to explore the effect of this parameter on the spectrum of the vertical structure operator, A. The number of vertical layers, $K$, is the other study parameter. The dominant modes are plotted in Figure 3 for $K=26$ and $K=96$. What can be seen in these solutions is that the modes are not well resolved, indeed, not converged, in the upper atmosphere with $K=26$. Since $p_{\text {top }}$ is small the modes are highly oscillatory near the top (upper atmosphere) boundary. This would correspond to the singular Strum-Liouville problem with a continuous spectrum.

The eigensolutions also reveal computational modes associated with the discretization. Looking at the smallest equivalent heights in Figure 4, it is clear that one is zero (or at least falls two orders of magnitude below the others). The vertical structure matrix $\mathbf{A}$ for the Lorentz vertical grid discretization contains one computational mode. This will be true regardless of the choice of the top and results from assignment of variables to the 

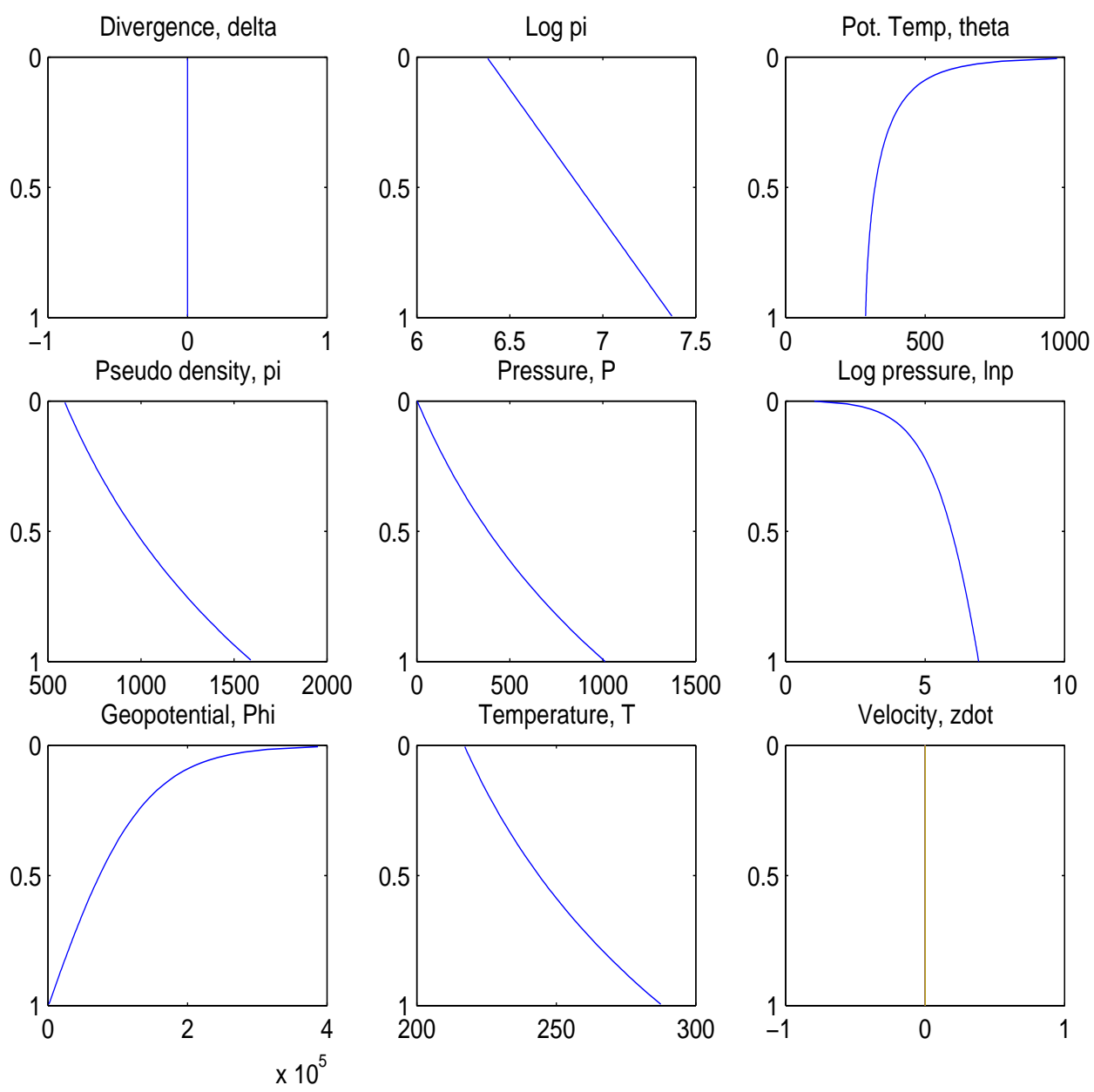

Figure 2: Lorentz Vertical Column representing a reference atmosphere with $p_{\text {top }}=2.8 \mathrm{hPa}$. 

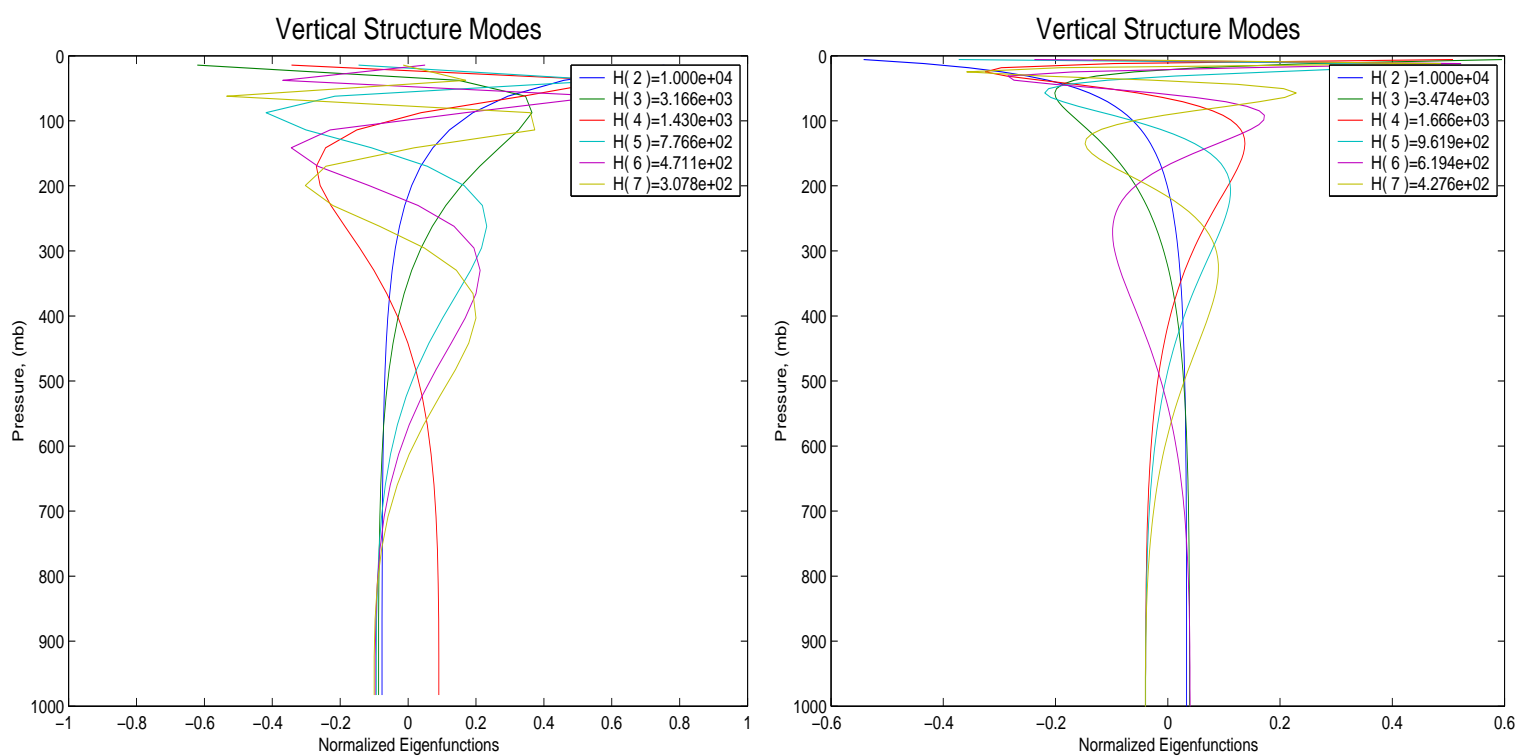

Figure 3: Normal modes of the vertical structure equation with $p_{\text {top }}=$ $2.9 \mathrm{hPa}$ and $K=26$ and 96 . Only the six largest modes (excluding the external mode), and their equivalent depths are displayed. Equivalent depths (m) are normalized so that the largest mode has a depth of $10 \mathrm{~km}$ 
grid and the required averaging that is required in the discretization. The computational mode associated with the zero equivalent height is plotted in Figure 5 along with the other nearby modes. It is associated with a surface value and can be controlled by eliminating this degree of freedom.

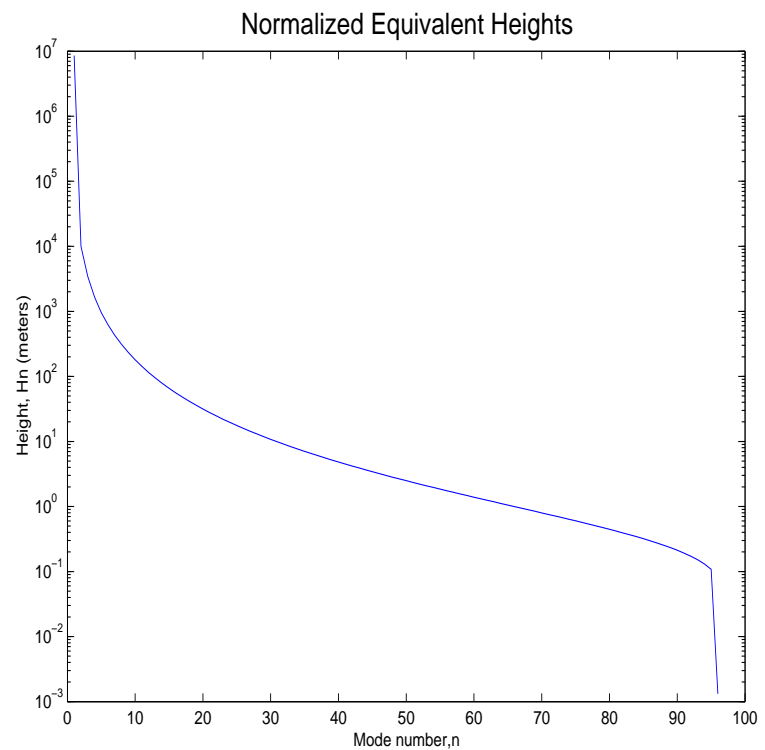

Figure 4: Equivalent depths for $p_{t o p}=2.9 \mathrm{hPa}$ and $K=96$.

Many other choices of the grid assignment are possible based on different formulations and vertical coordinate systems. The optimal choice for representation of the normal modes is considered in [32]. The Lorentz grid is found to generate errors in dispersion relations and to be suboptimal in terms of accuracy.

\subsection{Baroclinic Right Hand Sides}

With the data and methods available in the @lvgrid and @lvcolumn class the vertical terms of a baroclinic atmospheric model can be computed. In a semi-implicit treatment of the equations, such as in [10], the explicit terms are written on the right hand side of the equation. The method lvterms returns the right hand sides for the baroclinic equations.

The right hand side (explicit terms) of the momentum equation con- 


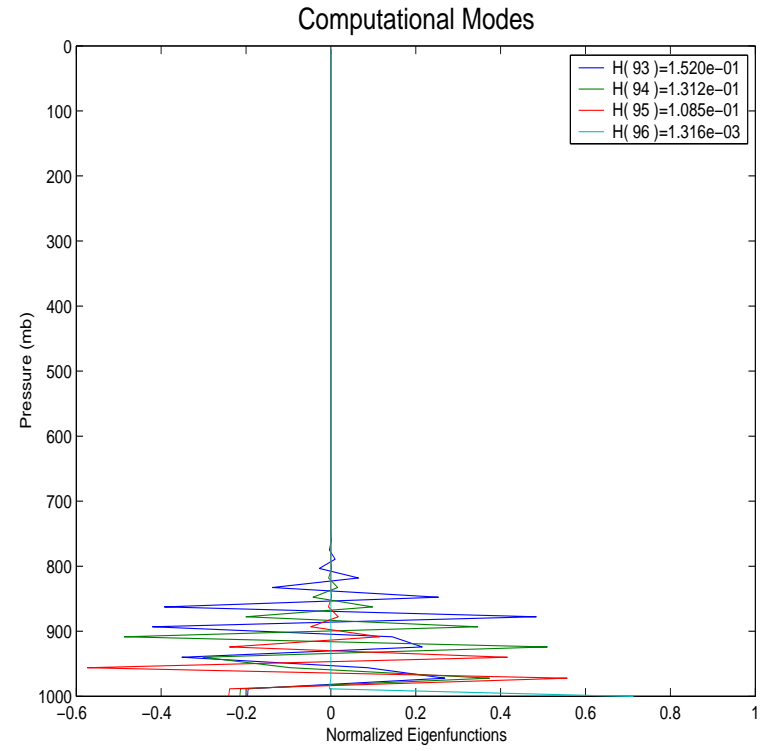

Figure 5: Computational Modes for the Lorentz grid $p_{\text {top }}=2.9 \mathrm{hPa}$ and $K=96$. 
tributing to the $R_{\eta}$ and $R_{\delta}$ (see [10]), is given by

$$
\mathbf{R}_{\mathbf{v}}=\mathbf{F}-\dot{\zeta} \frac{\partial \mathbf{v}}{\partial \zeta}+\left(R T \frac{\pi}{p}\left(\frac{\partial p}{\partial \pi}\right)\right)^{\prime} \nabla_{\zeta} \ln \pi
$$

The right hand sides of the continuity, energy and species equations are given by

$$
\begin{gathered}
R_{\pi}=-\frac{1}{\pi} \frac{\partial}{\partial \zeta}(\pi \dot{\zeta}) \\
R_{T}=\frac{Q}{C_{p}}-\dot{\zeta} \frac{\partial T}{\partial \zeta} \\
+\frac{\kappa T}{p}\left[\mathbf{v} \cdot\left(\pi\left(\frac{\partial p}{\partial \pi}\right) \nabla_{\zeta} \ln \pi\right)-\int_{\zeta_{t o p}}^{\zeta} \mathbf{v} \cdot\left(\pi \nabla_{\zeta} \ln \pi\right) d \zeta\right] \\
-\left(\frac{\kappa T}{p}\right)^{\prime} \int_{p_{t o p}}^{p(\zeta)} \delta d p \\
R_{\theta}=\frac{Q}{\Pi}-\dot{\zeta} \frac{\partial \theta}{\partial \zeta} \\
R_{q}=S-\dot{\zeta} \frac{\partial q}{\partial \zeta} .
\end{gathered}
$$

These right hand side terms can be used in the context of a baroclinic solution algorithm to produce a three dimensional atmospheric flow simulation. The algorithm demonstrated here is a semi-implicit, semi-lagrangian spectral algorithm. Though the equations are written quite generally to include hybrid sigma-pressure coordinates (as in [6]), isentropic or mass conserving vertical Lagrangian coordinates, the test code BCsigma.m uses a simple $\sigma$-coordinate. We take $\zeta=\frac{p-p_{t o p}}{p-p_{s}} \in[0,1]$ with uniform divisions.

The spectral solution of the semi-implicit equations for a baroclinic model follows [10].

\section{Conclusion}

Baroclinic, three diminsional models with the hydrostatic or non-hydrostatic assumption, exhibit a tight coupling of vertical and horizontal modes of motion. Since the vertical modes of the atmosphere are sensitive to the numerical treatment of the vertical grid, careful choice and study of the vertical discretization is important to the formulation of baroclinic models. A set of MATLAB routines for the Lorentz grid in a generalized vertical 
coordinate system has been described that computes the relevant vertical coupling terms of a baroclinic model. The underlying discretizations and MATLAB functions are tested with the solution of the vertical structure equation.

\section{Acknowledgments}

This report is one of a series concerning the use of numerical methods for global climate modeling. The work reported is sponsored by the Climate Change Prediction Program of the Office of Biological and Environmental Research and the Scientific Discovery Through Advanced Computing (SciDAC) program of the Office of Science, U.S. Department of Energy under contract number DE-AC05-00OR22750.

\section{A The 1976 Standard Atmosphere}

Conditions of the 1976 standard atmospheric column are used in this report. The equations that determine the standard atmosphere are those adopted 15 October 1976 by the United States Committee on Extension to the Standard Atmosphere (COESA). The equations and parameters used are documented in a book U.S. Standard Atmosphere, 1976 published by the U.S. Government Printing Office, Washington, D.C. A few values (Table 1) and a plot (Figure 6) are included here for reference.

\section{B Compact Vertical Discretization based on La- guerre Polynomials}

This appendix introduces a high order discretization of the vertical based on the use of a compact form for the derivatives, interpolations and quadratures required. We choose the vertical coordinate defined by $\zeta=-\ln p$. For this coordinate, the domain is nominally $p \in[0,1]$ with pressure at 1 atmosphere at the surface and zero going high above the atmosphere, so $\zeta \in[0, \infty)$ with $\zeta=0$ being the surface.

If the vertical grid points are chosen as the zeros of $L_{N}(\zeta)$, the Laguerre polynomial of degree $N$ and the abscissas of the Gauss-Laguerre quadrature method, then the integral term of interest from the hydrostatic equation is

$$
\int_{0}^{\zeta} e^{-\zeta} T d \zeta=\sum_{\zeta_{i} \leq \zeta} w_{i} T\left(\zeta_{i}\right)
$$




\begin{tabular}{||llll||}
\hline Pressure (hPa) & Height $(\mathrm{m})$ & Temperature $(\mathrm{C})$ & Layer \\
\hline 1000.0 & 110.88 & 14.28 & Boundary Layer \\
950.0 & 540.34 & 11.49 & Troposphere \\
900.0 & 988.50 & 8.57 & Troposphere \\
850.0 & 1457.30 & 5.53 & Troposphere \\
800.0 & 1948.99 & 2.33 & Troposphere \\
750.0 & 2466.23 & -1.03 & Troposphere \\
700.0 & 3012.18 & -4.58 & Troposphere \\
650.0 & 3590.69 & -8.34 & Troposphere \\
600.0 & 4206.43 & -12.34 & Troposphere \\
550.0 & 4865.22 & -16.62 & Troposphere \\
500.0 & 5574.44 & -21.23 & Troposphere \\
450.0 & 6343.62 & -26.23 & Troposphere \\
400.0 & 7185.44 & -31.71 & Troposphere \\
350.0 & 8117.27 & -37.76 & Troposphere \\
300.0 & 9163.96 & -44.57 & Troposphere \\
250.0 & 10362.95 & -52.36 & Troposphere \\
200.0 & 11784.05 & -56.50 & Tropopause \\
150.0 & 13608.42 & -56.50 & Tropopause \\
100.0 & 16179.72 & -56.50 & Tropopause \\
70.0 & 18441.62 & -56.50 & Tropopause \\
60.0 & 19419.19 & -56.50 & Tropopause \\
50.0 & 20576.17 & -55.92 & Stratosphere \\
20.0 & 26481.22 & -50.02 & Stratosphere \\
10.0 & 31054.64 & -45.45 & Stratosphere \\
5.0 & 35776.55 & -33.93 & Stratosphere \\
2.0 & 42439.85 & -15.27 & Stratosphere \\
1.0 & 47820.08 & -2.50 & Stratopause \\
0.7 & 50645.75 & -2.50 & Stratopause \\
0.6 & 51866.98 & -2.50 & Stratopause \\
0.5 & 53305.04 & -5.11 & Mesosphere \\
0.3 & 57253.58 & -13.01 & Mesosphere \\
0.1 & 65280.75 & -37.62 & Mesosphere \\
\hline
\end{tabular}

Table 1: The pressure, height and temperature of the 1976 Standard Atmosphere. Computed using stdatm76.f 


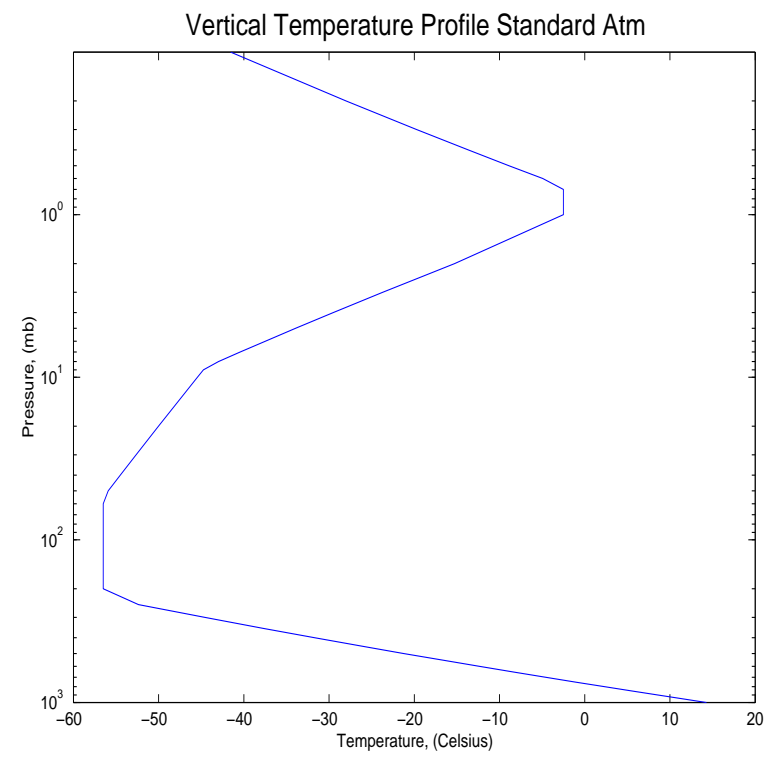

Figure 6: Standard Atmosphere Temperature Profile

The weights are given by the formula $w_{i}=\frac{\zeta_{i}}{(N+1)^{2}\left[L_{N+1}\left(\zeta_{i}\right)\right]^{2}}$. The integral will have an error term of $\frac{(N !)^{2}}{(2 N) !} T^{(2 N)}(\xi)$. But the Gauss-Laguerre points may not be a good choice for use in the atmosphere. The points in the troposphere are very few even with high order. For a 15 point rule, which will integrate a 29th degree polynomial exactly, there would be only 5 points in the troposphere. The advantage of using the very high order will be lost with points located too far from the surface. So we will chose the grid points arbitrarily and develop approximations that are exact with the Laguerre polynomials. The order of the methods will not have the Gauss quadrature advantage that obtains a doubling of the accuracy.

By a compact method for the derivative $[23,24]$ is meant an approximation of the form

$$
\sum_{j=L}^{M} A_{i j} u_{\zeta}\left(\zeta_{j}\right)=\sum_{j=J}^{K} B_{i j} u\left(\zeta_{j}\right) .
$$

The matrix $A$ and $B$ will be determined to get an accurate approximation about the point $x_{i}$. In terms of a stencil, the formula will use several implicit derivative points and several function value points. These do not need to be on the same grid, for example, staggered grids can be used with arbitrary 
locations. The compact procedure works for more than a derivative. We could use it for interpolation or quadrature, actually, an linear operator. To make this generalization, write the unknown point values as $\mathbf{d}$ and the known point values as $\mathbf{p}$. The compact approximation seeks matrices $\mathbf{A}$ and $\mathbf{B}$ such that $\mathbf{A d}-\mathbf{B p}=\mathbf{0}$,

$$
(\mathbf{D}, \mathbf{P})\left(\begin{array}{l}
\mathbf{A} \\
\mathbf{B}
\end{array}\right)=\mathbf{0},
$$

where $\mathbf{D}$ and $\mathbf{P}$ are Vandermonde matrices for $\mathbf{d}$ and $\mathbf{p}$. That is, we will have an exact approximation for the unknown for functions in a subspace of the larger space when evaluated at the grid points. The equation for the method coeffiecients evaluating derivatives $\left(\mathbf{d}=u_{\zeta}, \mathbf{p}=u\right)$ is

$$
\left(\begin{array}{cccccc}
1 & \ldots & 1 & 0 & \ldots & 0 \\
\mathbf{d}\left(L_{0}\right)\left(\zeta_{L}\right) & \ldots & \mathbf{d}\left(L_{0}\right)\left(\zeta_{M}\right) & -L_{0}\left(\zeta_{J}\right) & \ldots & -L_{0}\left(\zeta_{K}\right) \\
\cdot & \ldots & \cdot & \cdot & \ldots & \cdot \\
\mathbf{d}\left(L_{N}\right)\left(\zeta_{L}\right) & \ldots & \mathbf{d}\left(L_{N}\right)\left(\zeta_{M}\right) & -L_{N}\left(\zeta_{J}\right) & \ldots & -L_{N}\left(\zeta_{K}\right)
\end{array}\right)\left(\begin{array}{c}
A_{i}^{T} \\
B_{i}^{T}
\end{array}\right)=\left(\begin{array}{c}
1 \\
0 \\
\cdot \\
0
\end{array}\right)
$$

The first row is for normalization of the method coefficients, enforcing a sum of one.

The $\mathbf{A}$ and $\mathbf{B}$ can be chosen to give tridiagonal matrices if, for example, $L=i-1$ and $M=i+1$. Then the evaluation of unknowns involves a tridiagonal solve. In particular, $\mathbf{d}=\mathbf{A}^{-\mathbf{1}} \mathbf{B} \mathbf{p}$.

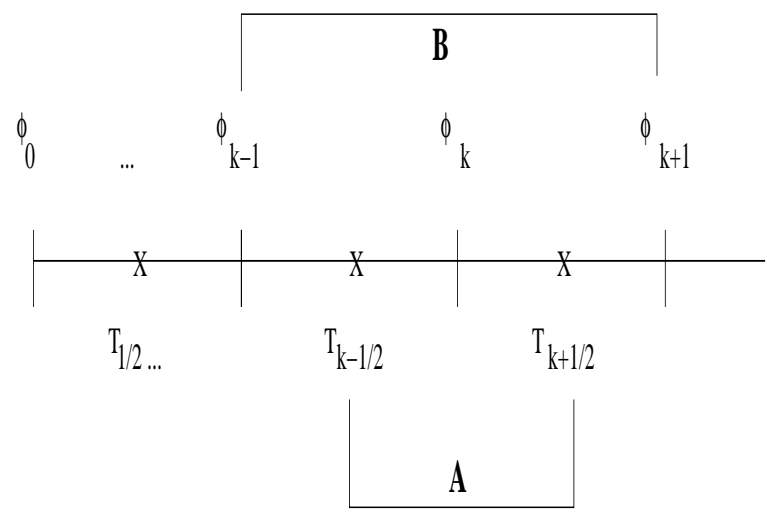

Figure 7: Stencil for Charney-Phillips grid with fourth order compact method. 
The hydrostatic equation can be approximated directly to solve for $\Phi$ from the vertical temperature profile. In the $\zeta$ coordinate system the hydrostatic equation is

$$
\Phi_{\zeta}=R T
$$

with $\Phi(0)=\Phi_{s}$. The compact approximation gives

$$
\mathbf{B} \Phi=R \mathbf{A} T .
$$

For the Lorentz grid, both $\Phi$ and $T$ are located at the integer levels so there is no staggering and the compact method is straightforward. For the Charney-Phillips grid, $T$ is located at half levels while $\Phi$ is located at integer levels. So the $\Phi_{\zeta}$ is needed at half levels (see Figure 7). The fourth order compact method on the Charney-Phillips grid would use 2 points for $\mathbf{A}$ and 3 points for $\mathbf{B}$ in order to maintain a tridiagonal solve for $\Phi$. At the surface boundary, this approximation is still natural with $\Phi(0)=\Phi_{s}$ imposed. Since the hydrostatic equation is a first order ordinary differential equation there is no boundary condition at $\infty$ for the hydrostatic equation. A finite truncation is still required. The points of evaluation of $\mathbf{d}\left(L_{n}\right)(\zeta)$ in the method equation (Eq. 39) are at the half levels. The hydrostatic matrices $H_{k l}$ can be formed from the matrices of the compact method using the relationship $\Phi=\Phi_{s}+R \mathbf{B}^{-1} \mathbf{A} T$, so that $\mathbf{H}=\mathbf{B}^{-1} \mathbf{A}$. In Figure 8, the geopotential solution is shown and the relative error with respect to a $K=1152$ solution is shown in Figure 9

The value of $p_{\text {top }}$ still acts as a regularization of the singular SturmLioville problem. Since the standard atmosphere temperature profile is only specified to a pressure of $0.10 \mathrm{mb}$, we examine the effect of setting $p_{t o p}=0.10$ as the most extreme upper boundary. (Extension of the temperature profile to infinity with a (bounded) value of absolute zero Kelvin, might be appropriate but we have not done this.) The accuracy is found to be comparable even in this case, showing that the numerical solution is insensitive to the choice of $p_{\text {top }}$. From this we conclude that the Laguerre polynomials that are used to construct the difference formulas, lend their asympotitc properties (bounded solution at infinity) to the computed solution.

Laguerre polynomials can be computed in MATLAB using the program LaguerrePoly(n) [31].

The vertical structure problem of the atmosphere can also be solved numerically using the compact method and we outline the solution formulation here. The equation in the $\zeta$-coordinate is

$$
\frac{d^{2} Z}{d \zeta^{2}}+\frac{R \gamma}{g H} e^{-\zeta} Z=0
$$




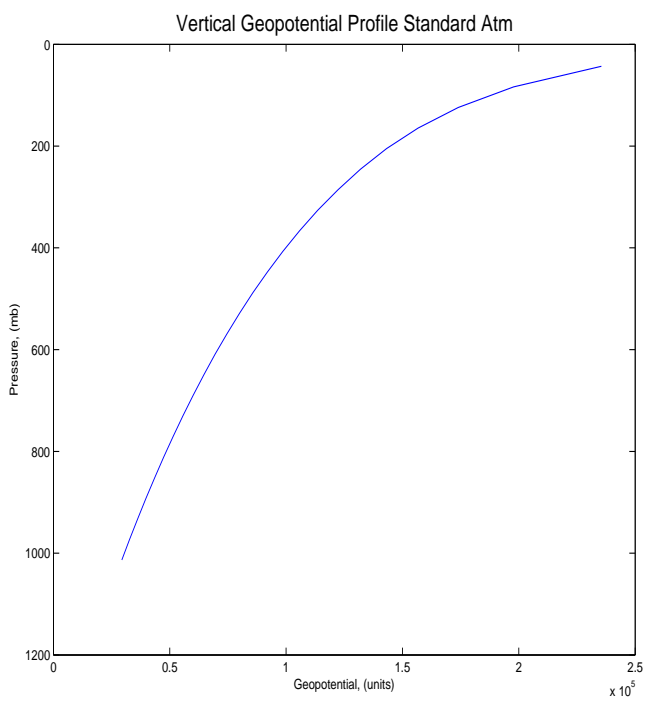

Figure 8: Hydrostatic solution for the geopotential using fourth order compact method $p_{\text {top }}=2.8 h P a$ and $K=26$. The standard atmosphere temperature profile is on a Charney-Phillips grid of equally spaced points in $\sigma$. 


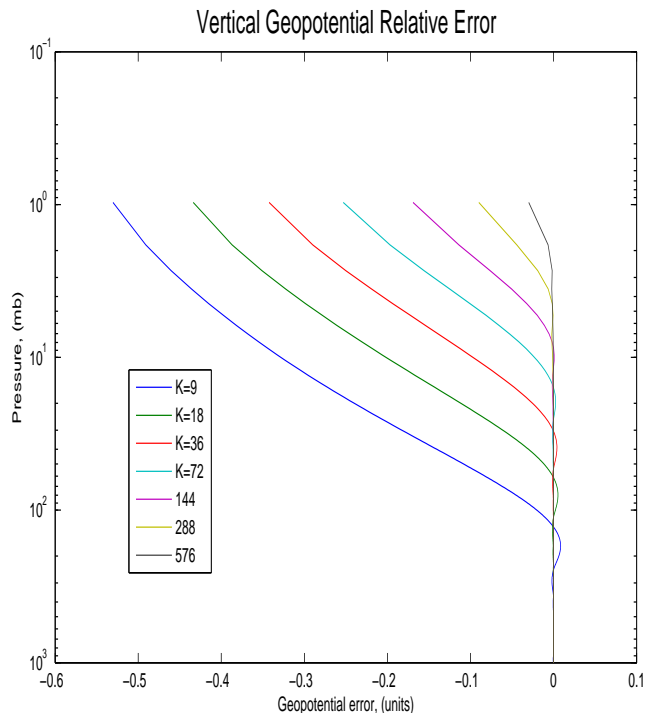

Figure 9: Relative error in geopotential $p_{t o p}=0.1 \mathrm{hPa}$ and $K=$ $9,18,36,72,144,288,576$ on a Charney-Phillips grid of equally spaced points in $\sigma$. 
with $e^{-\zeta} Z \rightarrow 0$ as $\zeta \rightarrow \infty$ and $\frac{d Z}{d \zeta}+\frac{\gamma}{T_{s}} Z=0$ at $\zeta=0$. The problem is transfered to the singular S-L problem on a semi-infinite interval, so we might expect the Laguerre polynomials and the associated weighted inner product to be of value in the approximation.

The compact method for the second derivative is set up with $\mathbf{d}=Z_{\zeta \zeta}$. The generalized eigenvalue problem for the vertical normal modes is

$$
(\mathbf{B}+\lambda \mathbf{A}) Z=0
$$

where the $e^{\zeta}$ has been absorbed into $\mathbf{B}$. The implementation of the boundary condition at $p_{\text {top }}$ is interesting. The fixed cap, flux boundary condition implemented for the Lorentz grid, is easily adapted to the Laguerre polynomials using the compact first derivative approximation. Since this is more restrictive than the bounded at infinity assumption, the interesting question is how much effect this has and whether another form of the boundary condition (eg. $\mathrm{Z}=$ arbitrary polynomial), would influence the normal modes.

The trouble with a spectral method in the vertical was discovered by Francis[13] and further elaborated in [28]. First, the number of GaussLaguerre points that fall in the physical range of the atmosphere is small requiring a large number of modes. As these modes are highly oscillatory in the top of the domain, a time step restriction on the dynamics is imposed and Francis found from a linear stability analysis that the stable time step is an order of magnitude smaller than the finite difference time step. Giving up on spectral accuracy with a set of discrete points not equal to the zeros of a Laguerre polynomial, but still using Laguerre as the basis functions for the compact discrete method, results in slow convergence to a solution as seen with the solution of the geopotential equation. This essentially rules out the Laguerre polynomial basis in favor of polynomials with more evenly distributed zeros. However, other polynomial basis may not have the desirable decay properties required for the singular boundary, i.e. $Z_{\zeta} \rightarrow 0$.

The solution adopted by the ECMWF for their semi-Lagrangian codes is a cubic finite element on a Charney-Phillips grid. This exploits superconvergence at the node points of the grid resulting in a sixth order scheme in the vertical [35]. This seems to be a very good solution, and indeed, they report significant reduction of noise in their simulations and improved forecast skill. With a Lagrangian coordinate, this would be like a moving finite element method. 


\section{References}

[1] K. Puri A. Kasahara. Spectral representation of three-dimensional global data by expansion in normal mode functions. Mon. Wea. Rev., 109, 1981.

[2] F Baer and J. Tribbia. On filtering of gravity modes through non-linear initialization. Mon. Wea. Rev., 105:1536-1539, 1977.

[3] R. Bleck. Numerical forecasting experiments based on the conservation of potential vorticity on isentropic surfaces. Appl. Meteor., 12:737-752, 1973.

[4] J.G. Charney. Geostrophic turbulence. J. Atmos. Sci., 28:1087-1095, 1971.

[5] E. Coddington and N. Levinson. Theory of Ordinary Differential Equations. McGraw Hill, New York, 1955.

[6] W.D. Collins, P.J. Rasch, B.A. Boville, J.J. Hack, J.R. McCaa, D.L. Williamson, B.P. Briegleb, C.M. Bitz, S.-J. Lin, and M. Zhang. The formulation and atmospheric simulation of the community atmosphere model version 3 (cam3). J. Climate, 19:2144-2161, 2005.

[7] W.D. Collins, P.J. Rasch, B.A. Boville, J.J. Hack, J.R. McCaa, D.L. Williamson, J.T. Kiehl, B.P. Briegleb, C. Bitz, S.-J. Lin, M. Zhang, and Y. Dai. Description of the ncar community atmosphere model (cam3.0). NCAR Techinical Note NCAR/TN-464+STR, NCAR, 2004.

[8] R. Daley. The development of efficient time integration schemes using model normal modes. Mon. Wea. Rev., 108:100-110, 1980.

[9] Roger Daley. Atmospheric Data Analysis. Cambridge University Press, Cambridge, 1991.

[10] J.B. Drake. Baroclinic dynamics with lagrange-semi-lagrangian treatment of advection. Mon. Wea. Rev., to be submitted, 2008.

[11] N. Wood E. Cordero, A. Staniforth. Normal mode analysis of the new dynamics. Numerical Weather Prediction Forecasting Research Tehenchical Report 393, U.K. MetOffice, 2002.

[12] A. Eliassen and E. Raustein. A numerical integration experiment with a model atmosphere based on isentropic coordinates. Meteor. Ann., 5:429-634, 1968. 
[13] P.E. Francis. The possible use of laguerre polynomials for representing the vertical structure of numerical models of the atmosphere. Quart. J. R. Met. Soc., 98:662-667, 1972.

[14] James R. Holton. An Introduction to Dynamic Meteorology. Academic Press, Inc., San Diego, second edition, 1979.

[15] D. R. Johnson. A generalized transport equation for use with meteorological coordinate systems. Mon. Wea. Rev., 108(6):733-745, 1980.

[16] D. R. Johnson, F. M Reames T. H. Zapotocny, and B.J. Wolf. A comparison of simulated precipitation by hybrid isentropic-sigma and sigma models. Mon. Wea. Rev., 121(7):2088-2114, 1993.

[17] Donald R. Johnson. The forcing and maintenance of global monsoonal circulations: An isentropic analysis. In Advances in Geophysics, volume 31, pages 43-316. 1987.

[18] D.R. Johnson. On the general coldness of climate models and the second law: Implications for modeling the earth system. J. Climate, to appear.

[19] A. Kasahara. Various vertical coordinate systems used for numerical weahter prediction. Mon. Wea. Rev., 102:509-522, 1974.

[20] A. Kasahara and J.H. Qian. Normal modes of a global nonhydrostatic atmospheric model. Mon. Wea. Rev., 128, 2000.

[21] W.T. Welch K.K. Tung. Remarks on charney's note on geostrophic turbulence. J. Atmos. Sci., 58:2009-2012, 2001.

[22] Celal S. Konor and Akio Arakawa. Design of an atmospheric model based on a generalized vertical coordinate. Mon. Wea. Rev., 125:16491673, 1997.

[23] S.K. Lele. Compact finite difference schemes with spectral-like resolution. J. Comp. Phys., 103:16-42, 1992.

[24] R.J. Purser Leslie, L.M. A comparative study of the perforamcne of various vertical discreization schemes. Meteorol. Atmos. Phys., 50:61$73,1992$.

[25] Shian-Jiann Lin. A vertically lagrangian finite-volume dynamical core for global models. Mon. Wea. Rev., 132:2293-2307, 2004. 
[26] Shian-Jiann Lin and Richard B. Rood. Multidimensional flux-form semi-lagrangian transport schemes. Mon. Wea. Rev., 124:2046-2070, 1996.

[27] B. Machenhauer. On the dynamics of gravity oscillations in a shallow water model with applications to normal mode initializiation. Contrib. Atmos. Phys., 50:253-271, 1977.

[28] A. Mizzi, J. Tribbia, and J. Curry. Vertical spectral representation in primitive equation models of the atmosphere. Mon. Wea. Rev., 123:2426-2446, 1995.

[29] Rick Salmon. Lectures on Geophysical Fluid Dynamics. Oxford University Press, Oxford, first edition, 1998.

[30] Masaki Satoh. Atmopsheric Circulation Dynamics and General Circulation Models. Springer-Verlag, Berlin, 2004.

[31] David Terr. Laguerrepoly.m: Coefficients of the laguerre polynomial $l_{n}$, given $n$. MATLAB Central File Exchange, ( http://www.mathworks.com/matlabcentral ), 2004.

[32] J. Thuburn and T.J. Woolings. Vertical discretizations for compressible euler equation atmospheric models giving optimal representation of normal modes. J. Comp. Phys., 203:386-404, 2005.

[33] R. D. Townsend and D. R. Johnson. A diagnostic sutdy of the isentropic zonally averaged mass circulation during the first GARP blobal experiment. J. Atmos. Sci., 42(15):1565-1579, 1985.

[34] L.W. Uccellini, D. R. Johnson, and R. E. Schlesinger. An isentropic and sigma coordinate hybrid numerical model: Model devlopment and some initial tests. J. Atmos. Sci., 36(3):390-414, 1979.

[35] A. Untch and M. Hortal. A finite element scheme for the vertical discretization of the semi-lagrangian version of the ecmwf forecast model. Q. J. R. Meteorol. Soc., 130:1505-1530, 2004.

[36] L.P. Chang Y.K. Sasaki. Numerical solution of the vertical structure equation in the normal mode method. Mon. Wea. Rev., 113, 1985.

[37] T. H. Zapotocny, A.J. Lenzen, D.R. Johnson, T.K Schaack, and F. M Reames. A comparison of inert trace constituent transport between the university of wisconsin isentropic-sigma model and the NCAR Communtiy Climate Model. Mon. Wea. Rev., 121(7):2088-2114, 1993. 